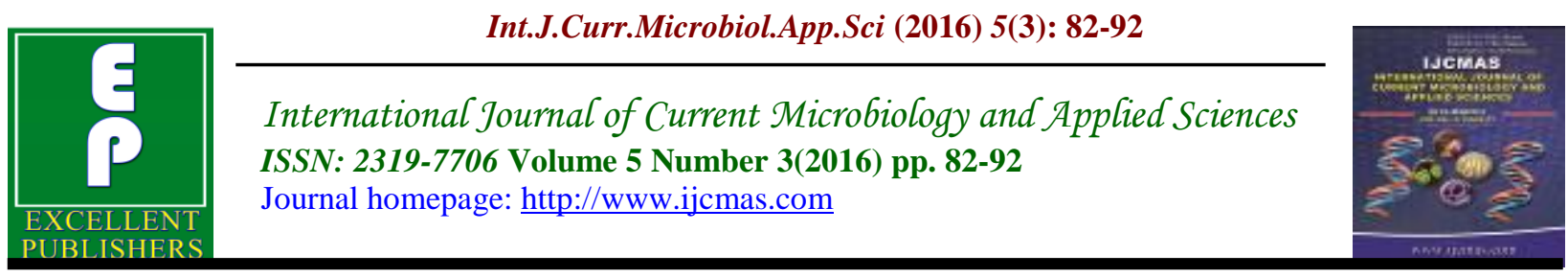

Original Research Article

http://dx.doi.org/10.20546/ijcmas.2016.503.012

\title{
Aquatic and Semi Aquatic Ornamental Flora of Karimnagar District, Telangana, India
}

\author{
G. Odelu* \\ Department of Botany, Government Degree College, Jammikunta, Karimnagar, \\ (Satavahana University) Telangana-50512, India \\ *Corresponding author
}

\begin{abstract}
A B S T R A C T
Keywords

Endangered, Floristic survey, IUCN, Ornamental plants, Pollution.

Article Info

Accepted:

08 February 2016

Available Online:

10, March 2016

Commercial crops are very well-known verities coming from result of cross breeding with wild species. A variety of wild survival of many is endangered by over exploitation by plants are highly useful to the local people, while the human beings. Ornamental plants improperly placed in relation to the pollution, social and rural forestry, and wasteland conformation of the land, roads and buildings. The total enumerated plants are 80 species, 65 genera belonging to 38 families. In this 80 species 55 from dicots, 23 from monocotyledons and 2 pteridophytes.Monospecious families are 20. Highest number of species from Asteraceae(10),followed by Cyperaceae (08), Fabaceae(04). Categories they are free floating(FF), submerged with anchoring(SA), rooted anchoring(RA), emergent and anchoring (EA)and floating submerged with anchoring(FSA).
\end{abstract}

\section{Introduction}

Wild plants are genetic resources for new verities, cultivars in all aspects of human being. Based on human population needs for food i.e. Rice, wheat, maize, jowar etc (1). Commercial crops are very well-known verities coming from result of cross breeding with wild species. Even resistant crop verities and qualitative characters coming from wild species. $(2,3)$ Including that plants which are known as ornamental plants also produced basically from wild species, unfortunately many of them have been destroyed, they show great variation in composition and density in marked to such an extent that several have become extinct and contrast with domesticated plants. A variety of wild survival of many is endangered by over exploitation by plants are highly useful to the local people, while the human beings. Even forest area s also focused only which are commercial important. Wild flora Ornamental plants which have special are very important in view of aesthetic and recreational structures on a value for man. Present scenario of these plants is day by days critical to survive as natural habitats. (4) 
Due to the anthropogenic activities caused to rapidly undergo to urbanisation, industrialisation and elevates levels of pollution everywhere.(4) There is no place for people to spend some time in natural aesthetic and swallow of beauty of nature. So some of the cities town's even big villages also planned for to make gardens, public gardens, in conservation manner government and N.G.O. Organisations supported to this programme. Flowers have the calibre to produce fragrance. Some of them plants support, they are annual or perennials.(5) These are produced from wild progenitor, few of which still exist in natural add beauty to the garden due to attractive flowers of habitat. A large number naturally in the field and have highly ornamental features of ornamental plants are available which can be used to such as ornamental flowers, foliage and fruits. the ornamental plants improperly placed in relation to the pollution, social and rural forestry, wasteland conformation of the land, roads and buildings. They using mainly in landscaping of outdoor, some special type of plants are in indoor spaces. $(6,7)$ Water is the main source of to carry the lives of every organism. Some of the attractive flower bearing plants is adapted to aquatic habitat and some are semi aquatic.(8) Present research work also deals with the ornaments which are grow in aquatic, semi aquatic, and wet places. Earlier workers also give the importance of this topic. This floristic survey undertaken in this area is first time, earlier A. M. Naqui work on mainly on native flora of Karimnagar district entitled with 'Flora of Karimnagar".(9).

\section{Study Area}

Study area belonging to Telangana state, which is newly formed $29^{\text {th }}$ state in our India. Study area bordered with west side Nizamabad, North Adilabad, East with
Warangal, south bordered with Medak district of Telangana. Karimnagar atmospherically inland climatic conditions, gets most of its rainfall from the Southwest monsoon, during this period temperatures range from a minimum of $27^{\circ} \mathrm{C}$ to a maximum of $39{ }^{\circ} \mathrm{C}$. The highest recorded is around $44{ }^{\circ} \mathrm{C}$. Humidity is around $50 \%$. October and November experiences increased rainfall from the Northeast monsoon. During this time, daytime temperatures average around $30{ }^{\circ} \mathrm{C}$. The winter season starts in December and lasts through February. During this time, temperatures range from a minimum of $20{ }^{\circ} \mathrm{C}$ to a maximum of $35^{\circ} \mathrm{C}$.

Latitude 1705'0" N, Longitude 78029'0" E, Altitude $1600 \mathrm{M}$ forest area is 2, 50,400 ha. Districts forest divided to two divisions they are Karimnagar West with 9 Beats, Karimnagar East with 27 Beats.

\section{Materials and Methods}

The main objective of research collection to study the whole aquatic and semi aquatic plants. Research part divided into two parts one is observation, collection of plans and identification from various urban and rural ornamental plants, secondly go to forest area collection of which species and compare them. Through field work from selected sites of public gardens, water bodies which are located in parks.

This work carry from 2012 June-2015 April. The collected taxa used for preparation of Herbaria, preserved in department of Botany, GDC with voucher numbers. We can identify with the help of taxonomy literature, The Flora of Madras Presidency by Gamble,(10,11 ) Flora of Andhra Pradesh by Prof. T. Pullaiah,(12,13) experts and internet facility. Preparation of samples by according to Jain and Rao 1977 (14). 


\section{Results and Discussion}

Present study indicates that the wild flora can be exploited to urban areas. Present scenario of some reserve forest is in critical conditions due the policies of the government. The total enumerated plants are 80 species, 65 genera belonging to 38families. In this 80 species, 55 from Dicots, 23 from Monocotyledons and 2 Pteridophytes. Monospecious families are 20. Genera are from dicots 46, Monocots 17, Pteridophytes are 02. Families from dicots are 25, monocots 11, 2families from pteridophytes. Highest number of species from Asteraceae (10), followed by Cyperaceae (08), Fabaceae (04).
Concern with their habitat several researchers divided the aquatic to mainly four types they are Free Floating, Submerged with Anchoring, Rooted Anchoring, Emergent and Anchoring (15). But there are several confusing classifications also there by their flexibility of the aquatic habitats and morphological forms of plants. Present study classified them under five categories they are free floating (FF), submerged with anchoring (SA), rooted anchoring (RA), emergent and anchoring (EA) and floating submerged with anchoring(FSA). They are given in Fig. No 1.

Table.1 Documented Ornamental Flora

\begin{tabular}{|l|l|l|l|l|}
\hline S.No & Scientific name of the plant & Family & Habitat & $\begin{array}{l}\text { IUCN 2015 } \\
\text { Ver.3.1 }\end{array}$ \\
\hline 1 & Aeschynomene aspera L & Fabaceae & EA & LC \\
\hline 2 & Ageratum conyzoides & Asteraceae & EA & NE \\
\hline 3 & Alisma plantago-aquatica & Alismataceae & FSA & LC \\
\hline 4 & Alternanthera philoxeroides (Mart.) Griseb. & Amaranthaceae & EA & LC \\
\hline 5 & Aponogeton natans & Aponogetonaceae & FSA & LC \\
\hline 6 & Argyreia pilosa Arn. & Convolvulaceae & EA & LC \\
\hline 7 & Azollaflucoidis cristata & Salviniaceae & FF & NE \\
\hline 8 & Bacopa monnieri & Scrophulariaceae & SA & LC \\
\hline 9 & Blumea mollis & Asteraceae & EA & NE \\
\hline 10 & Boerhavia chinensis (L.) & Nyctaginaceae & EA & DD \\
\hline 11 & Canna indica & Cannaceae & SA & LC \\
\hline 12 & Celosia argentea L. & Amaranthaceae & EA & NE \\
\hline 13 & Chamaecrista pumila & Caesalpinaceae & EA & NE \\
\hline 14 & Chromolaena odorata & Asteraceae & EA & NE \\
\hline 15 & Chrysopogon aciculatus & Poaceae & EA & NE \\
\hline 16 & Colocasia esculentus & Ararceae & SA & DD \\
\hline 17 & Crotalaria pallida & Fabaceae & EA & NE \\
\hline 18 & Cyanotis axillaris & Commelinaceae & SA & LC \\
\hline 19 & Cyanotis cristata & Commelinaceae & SA & LC \\
\hline 20 & Cynodon dactylon & Poaceae & EA & NE \\
\hline 21 & Cyperus acuminatus & Cyperaceae & EA & LC \\
\hline 22 & C.alterni Folious & Cyperaceae & EA & DD \\
\hline 23 & C.erythrorhizos & Cyperaceae & EA & LC \\
\hline 24 & C. esculentus & Cyperaceae & EA & LC \\
\hline 25 & C. rotundus & Cyperaceae & EA & LC \\
\hline & & & & \\
\hline
\end{tabular}




\begin{tabular}{|c|c|c|c|c|}
\hline 26 & C. strigosus & Cyperaceae & EA & LC \\
\hline 27 & Eclipta alba & Asteraceae & EA & $\mathrm{DD}$ \\
\hline 28 & E. prostrata & Asteraceae & EA & DD \\
\hline 29 & Eichhornia crassipes (Mart & Pontederiaceae & FF & $\mathrm{NE}$ \\
\hline 30 & Emilia sonchifolia (L.) & Asteraceae & EA & DD \\
\hline 31 & Glinus lotoidesL & Molluginaceae & EA & DD \\
\hline 32 & Gloriosa superba L. & Liliaceae & EA & VU \\
\hline 33 & Grangea maderaspatana & Asteraceae & EA & $\mathrm{LC}$ \\
\hline 34 & Hedyotis auricularia & Rubiaceae & EA & $\mathrm{NE}$ \\
\hline 35 & Hedyotis verticillata & Rubiaceae & EA & $\mathrm{NE}$ \\
\hline 36 & Heliotropium curassavicum $L$ & Boraginaceae & EA & LC \\
\hline 37 & H.indicum & Boraginaceae & EA & LC \\
\hline 38 & Hydrolea zeylanica & Hydrophyllaceae & FSA & LC \\
\hline 39 & Hygrophila auriculata & Acanthaceae & SA & LC \\
\hline 40 & IIlysanthes rotundifolia & Linderniaceae & FSA & $\mathrm{NE}$ \\
\hline 41 & Ipomoea aquatica & Convolvulaceae & FSA & LC \\
\hline 42 & I.carnea & Convolvulaceae & FSA & LC \\
\hline 43 & I.hederifolia $L$ & Convolvulaceae & FSA & $\mathrm{LC}$ \\
\hline 44 & Justicia betonica & Acanthaceae & EA & DD \\
\hline 45 & Lindenbergia indica & Scrophulariaceae & FSA & LC \\
\hline 46 & Ludwigia octavalvis & Onagraceae & SA & $\mathrm{NE}$ \\
\hline 47 & L. peruviana (L.) & Onagraceae & EA & $\mathrm{NE}$ \\
\hline 48 & Marsilia quadrifolia & Marsiliaceae & SA & $\mathrm{LC}$ \\
\hline 49 & Melochia corchorifolia $L$ & Sterculiaceae & EA & $\mathrm{NE}$ \\
\hline 50 & Merremia dissecta (Jacq.) Hall. F. & Convolvulaceae & EA & $\mathrm{NE}$ \\
\hline 51 & M. Quinquefolia (L.) Hallier F. & Convolvulaceae & EA & $\mathrm{NE}$ \\
\hline 52 & Mikania Micrantha & Asteraceae & EA & LC \\
\hline 53 & Mollugo. nudicaulis & Molluginaceae & EA & DD \\
\hline 54 & M.pentaphylla & Molluginaceae & EA & DD \\
\hline 55 & Monochoria viginalis (Burm. F.) Presl, Reliq. & Pontoridaceae & FF & $\mathrm{NE}$ \\
\hline 56 & Myriophyylum indicum & Haloragaceae & FSA & LC \\
\hline 57 & Nelumbo nucifera Gaertner & Nelumbonaceae & RA & $\mathrm{NE}$ \\
\hline 58 & Nymphaea alba & Nymphaceae & RA & LC \\
\hline 59 & N. pubescens Willd & Nymphaceae & RA & LC \\
\hline 60 & Nymphoidis cristata & Nymphaceae & RA & LC \\
\hline 61 & Oldenlandia corymbosa & Rubiaceae & EA & LC \\
\hline 62 & Ottelia alismoides (L.) Pers & Hydrocharitaceae & RA & $\mathrm{NE}$ \\
\hline 63 & Oxalis aorniculata $L$. & Oxallidaceae & EA & LC \\
\hline 64 & Oxystelma esculentum $(L$. & Apocynaceae & EA & LC \\
\hline 65 & Pergularia daemia (Forsskal) & Ascepiadaceae & EA & DD \\
\hline 66 & Persicaria glabra (Willd.) M. Gomez & Polygoniaceae & SA & DD \\
\hline 67 & Pistia stratoites L. & Ararceae & FF & LC \\
\hline 68 & Polygonum puberscens Blume & Polygoniaceae & SA & DD \\
\hline 69 & Phyla nodiflora (L.) Greene & Verbinaceae & EA & DD \\
\hline 70 & Physalis minima L. & Solanaceae & EA & DD \\
\hline 71 & Sagittaria latifolia Willd & Alismataceae & SA & DD \\
\hline 72 & Scirpus atrovirens & Cyperaceae & EA & $\mathrm{NE}$ \\
\hline 73 & S.s cyperinus & Cyperaceae & EA & $\mathrm{NE}$ \\
\hline 74 & Spaeraranthus indica & Asteraceae & EA & $\mathrm{NE}$ \\
\hline
\end{tabular}




\begin{tabular}{|l|l|l|l|l|}
\hline 75 & Synedrella nodiflora & Asteraceae & EA & DD \\
\hline 76 & Tephrosia pumila & Fabaceae & EA & NE \\
\hline 77 & Thunbergia fragrans Roxb & Acanthaceae & EA & DD \\
\hline 78 & Typha angustifolia & Typhaceae & SA & LC \\
\hline 79 & Urena lobata L. & Malvaceae & EA & LC \\
\hline 80 & Vigna trilobata & Fabaceae & EA & NE \\
\hline
\end{tabular}

Figure.1

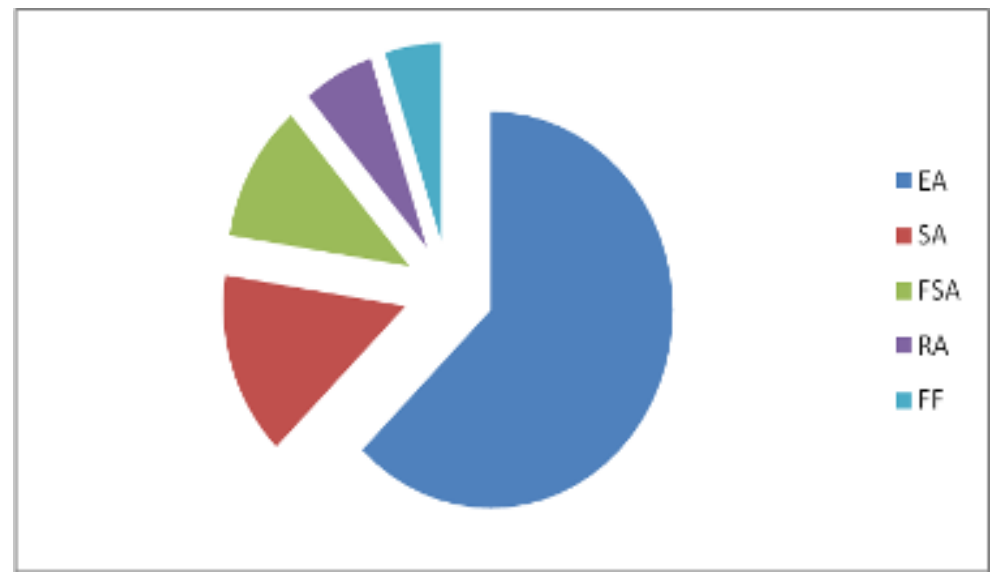

Figure.2

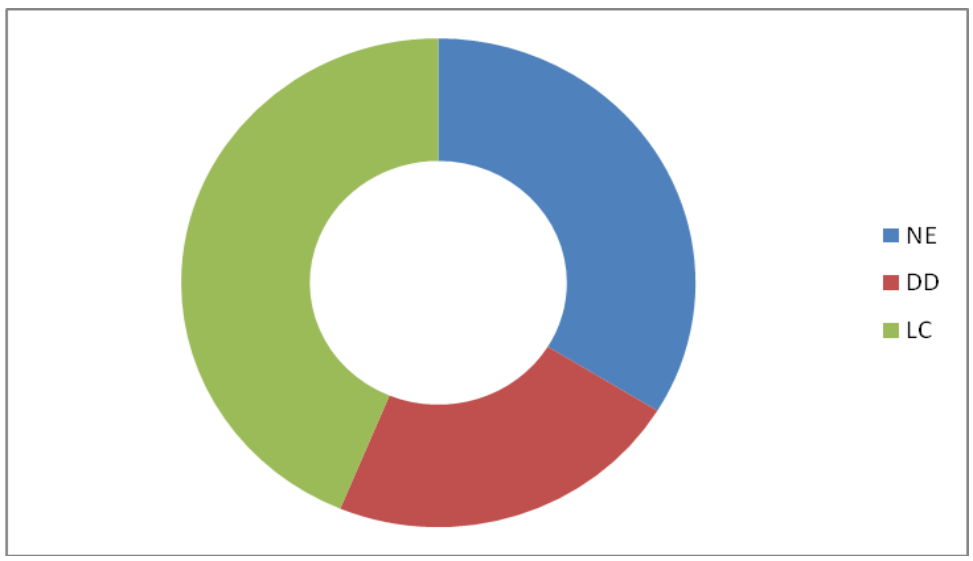


Plate.1

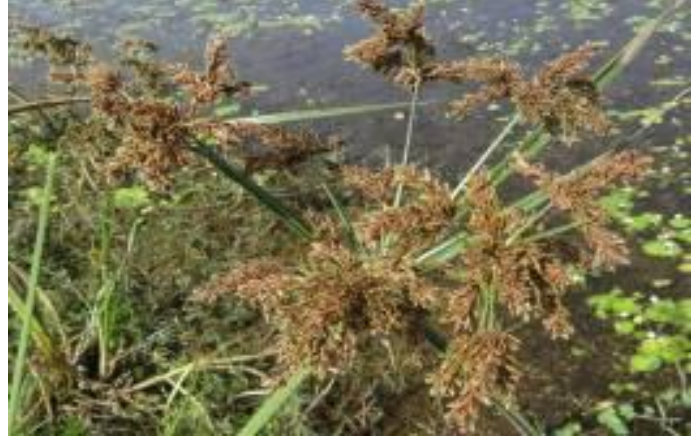

1) Cyperus strigosus

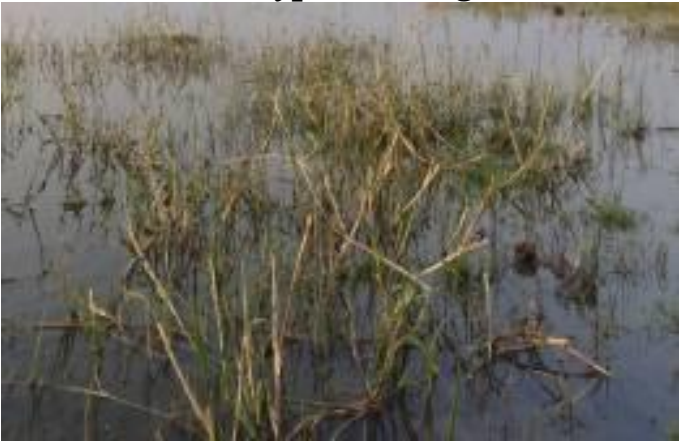

3) Hydrolea zeylanica

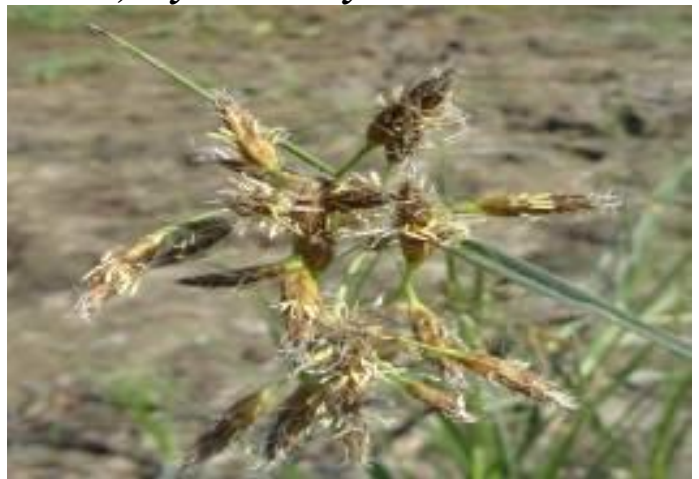

5) Cyperus erythrorhizos

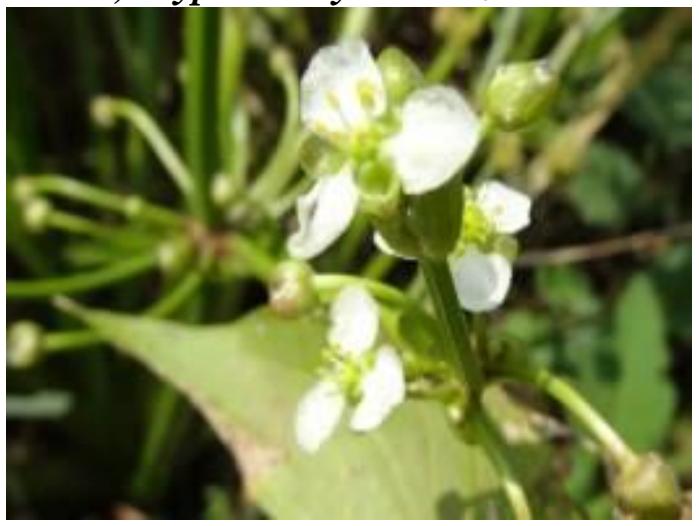

7) Alisma plantago-aquatica

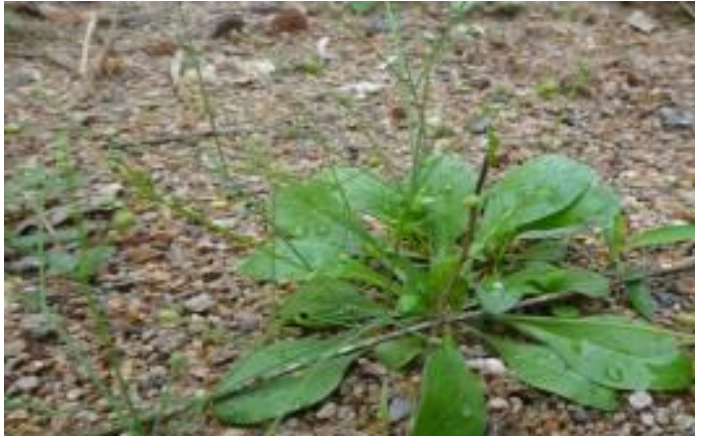

2) M. nudicaulis

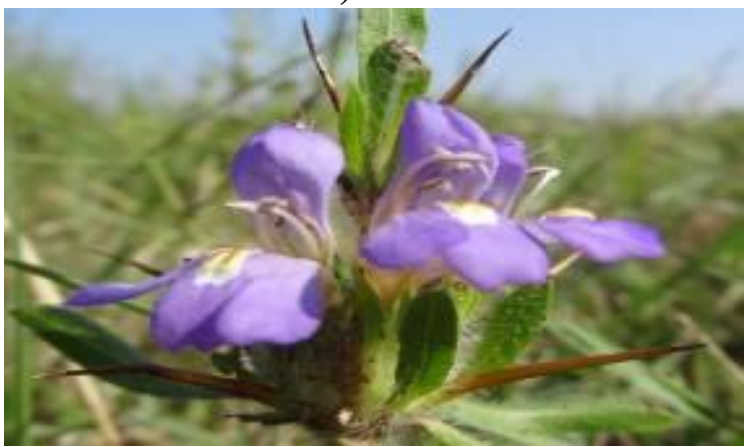

4) Hygrophila auriculata

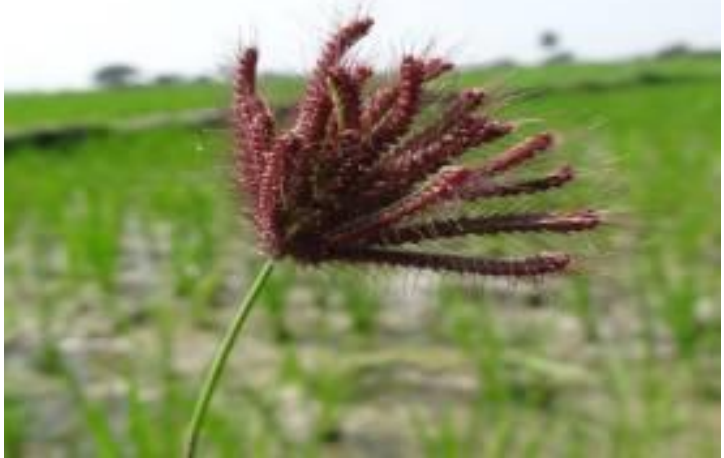

6) Chrysopogon aciculatus

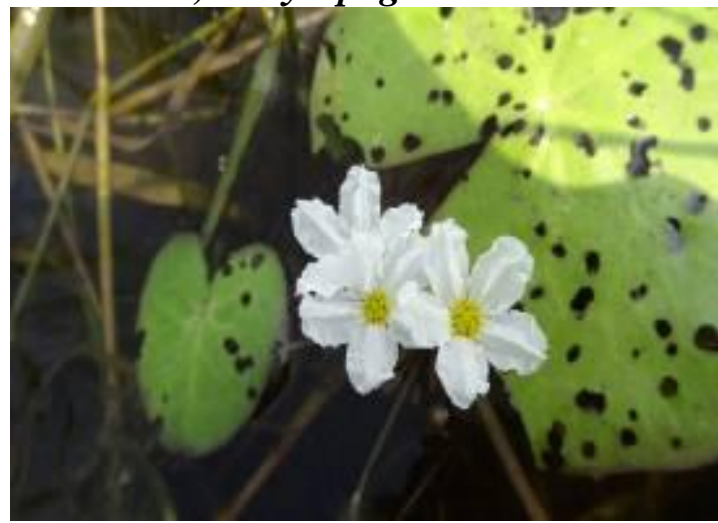

8) Nymphoidis cristata 
Plate.2

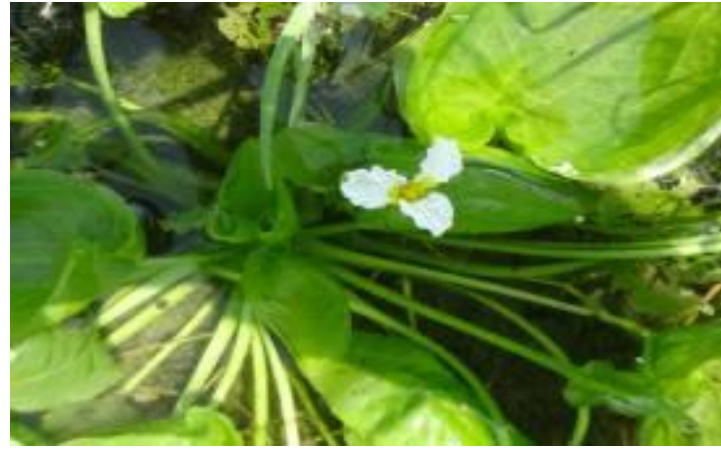

9) Ottelia alismoides (L.) Pers

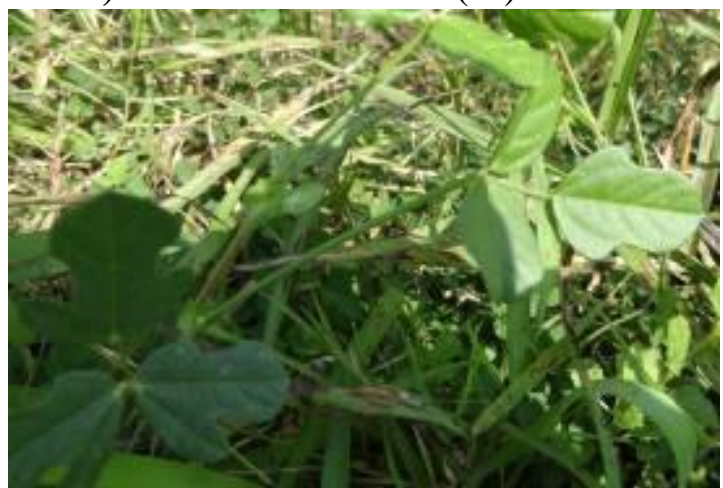

11) Vigna trilobata

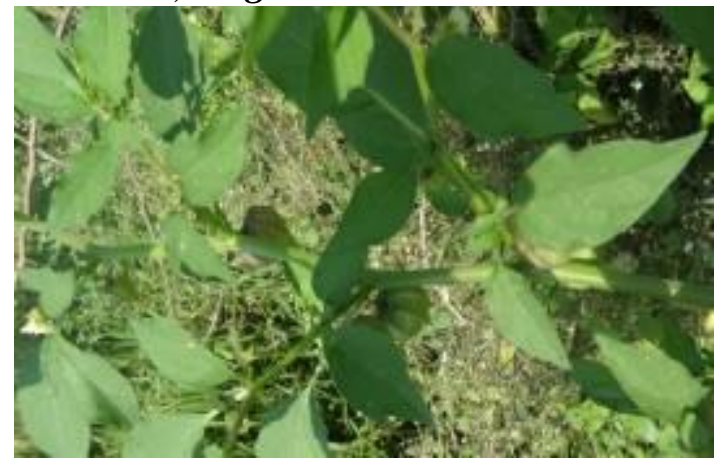

13) Physalis minima $L$.

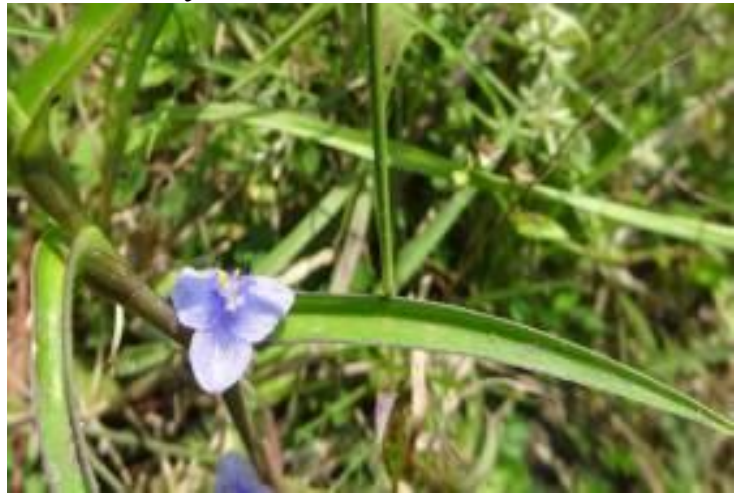

15) Cyanotis cristata

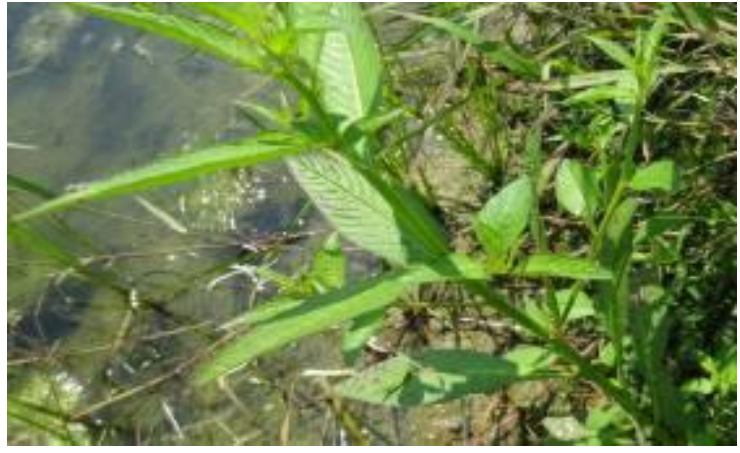

10) Ludwigia peruviana (L.)

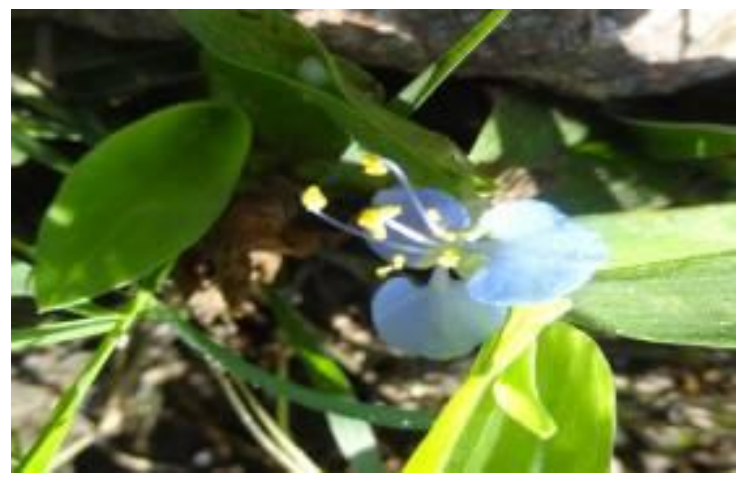

12) Cyanotis axillaris

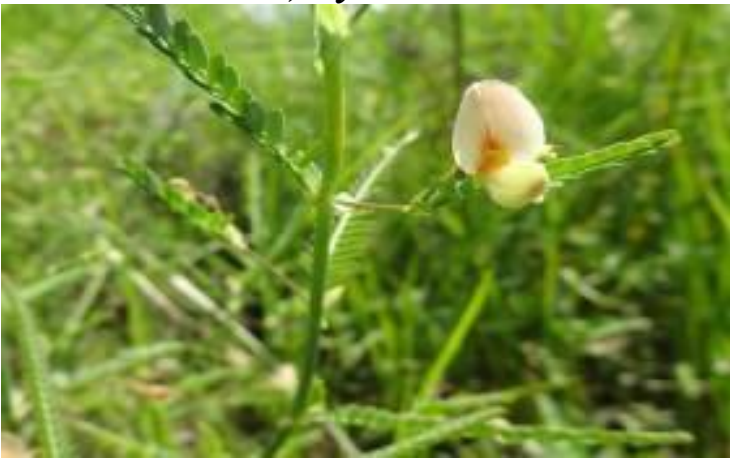

14) Aeschynomene aspera $L$

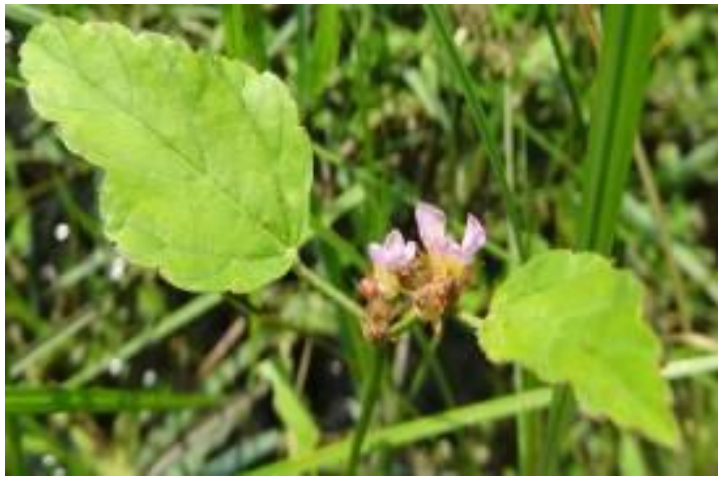

16) Melochia corchorifolia $L$ 
Plate.3

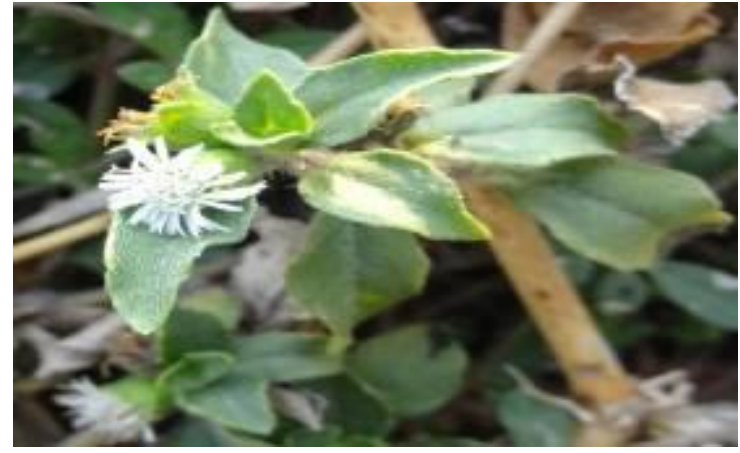

17) Eclipta alba

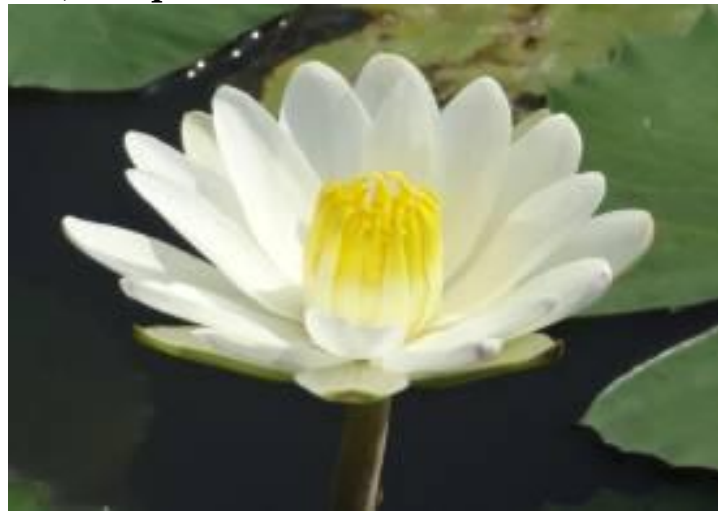

19) Nymphaea alba

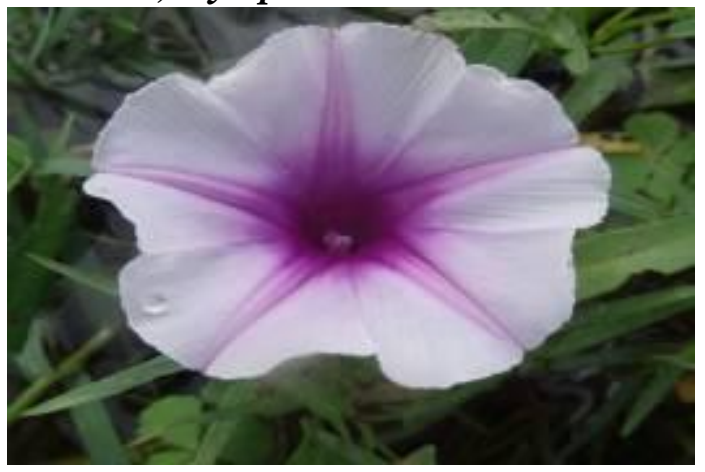

21) Ipomoea aquatica

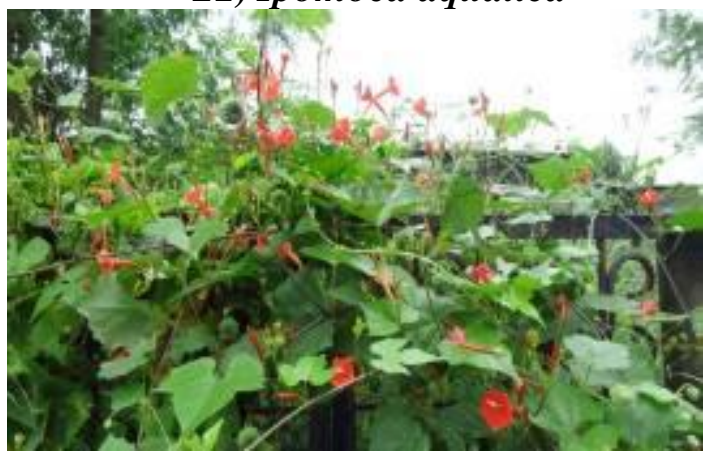

23) I.hederifolia $L$

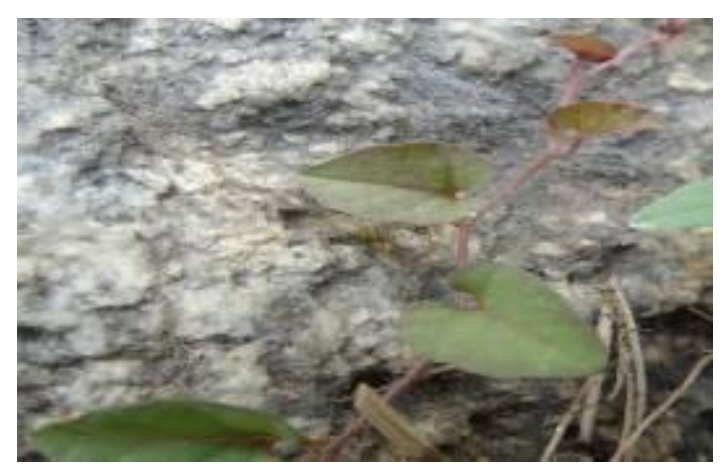

18) Merremia dissecta (Jacq.) Hall. F.

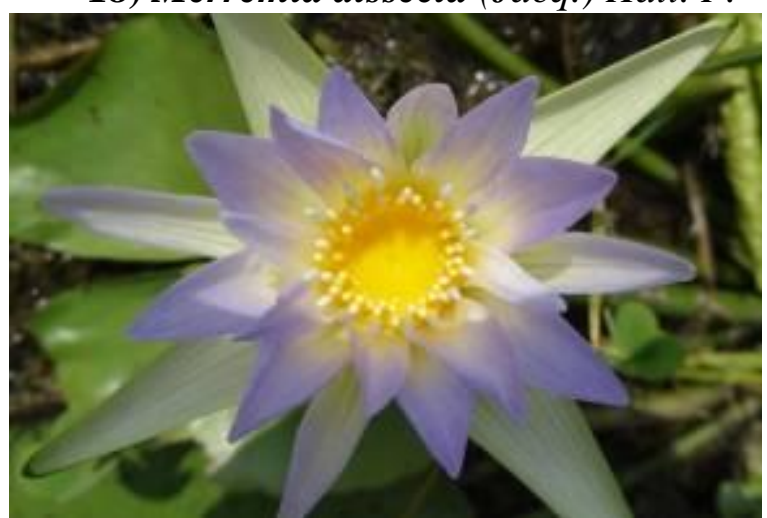

20) N.pubescens Willd

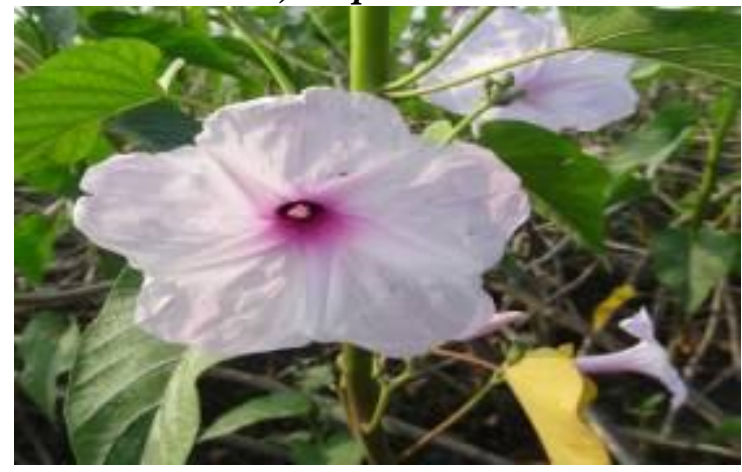

22) I.carnea

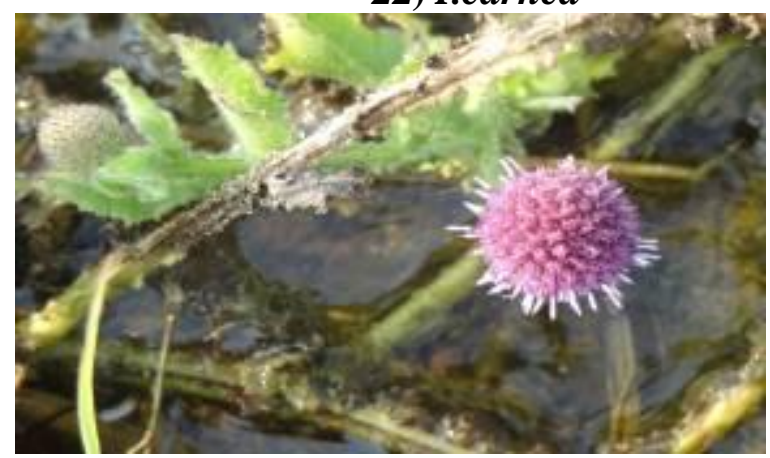

24) Spaeraranthus indica 
Plate.4

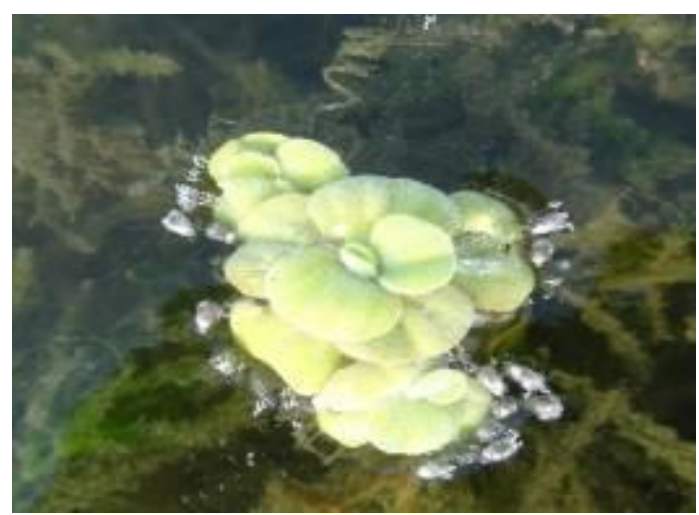

25) Pistia stratoites $L$.

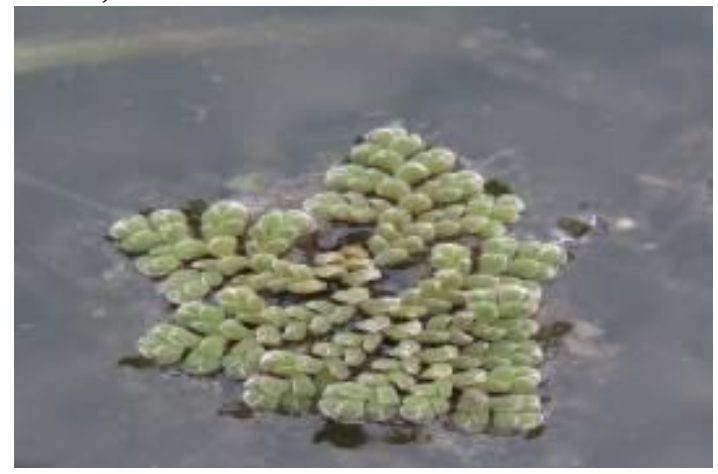

27) Azollaflucoidis cristata

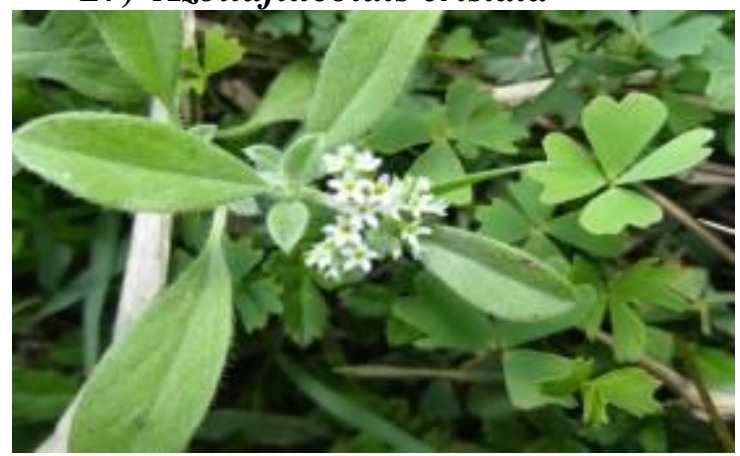

29)Heliotropium curassavicum $L$

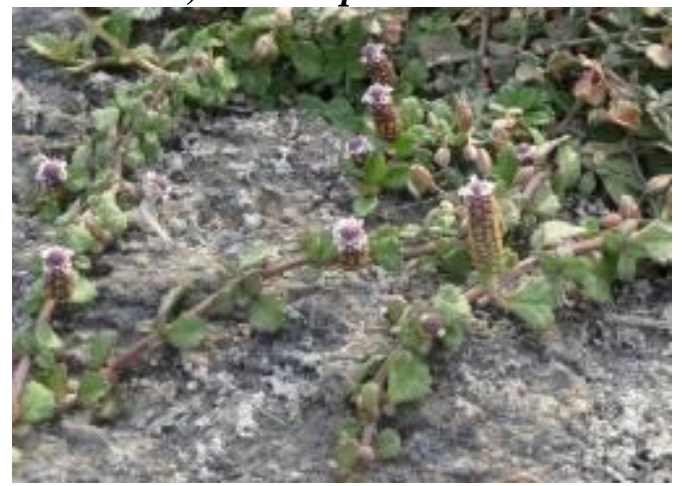

31) Phyla nodiflora (L.) Greene

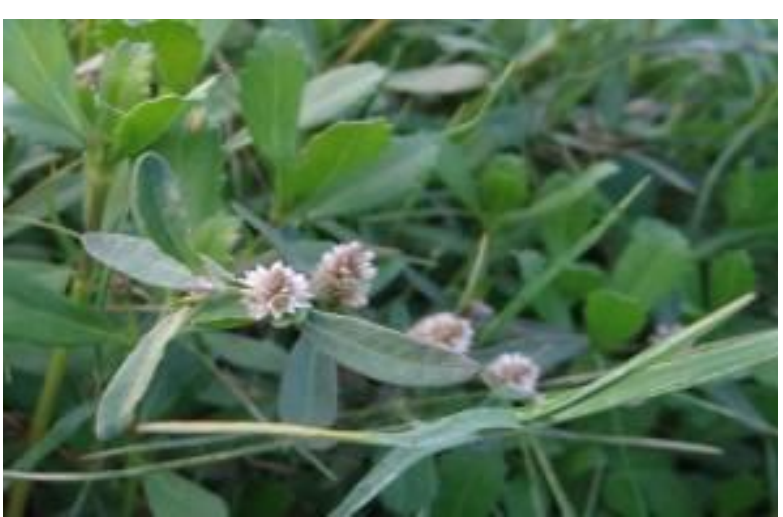

26) Alternanthera philoxeroides (Mart.) Griseb.

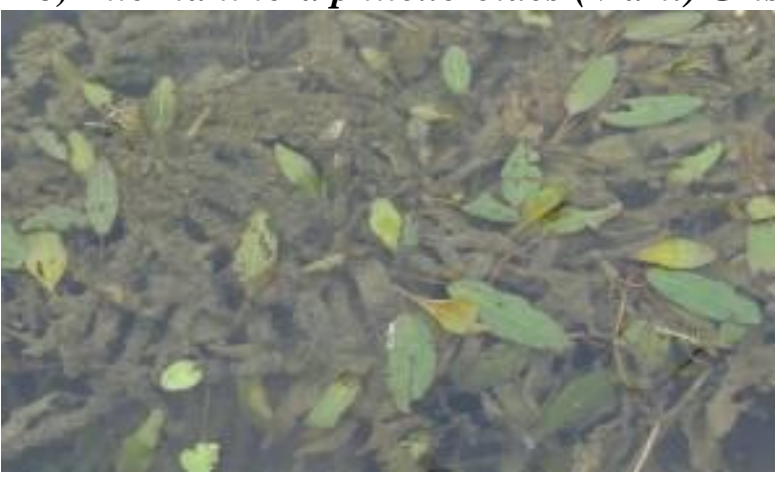

28) Aponogeton natans

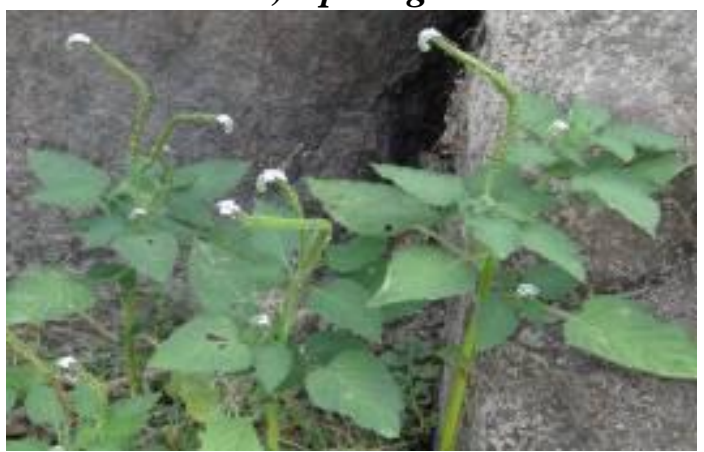

30) H.indicum

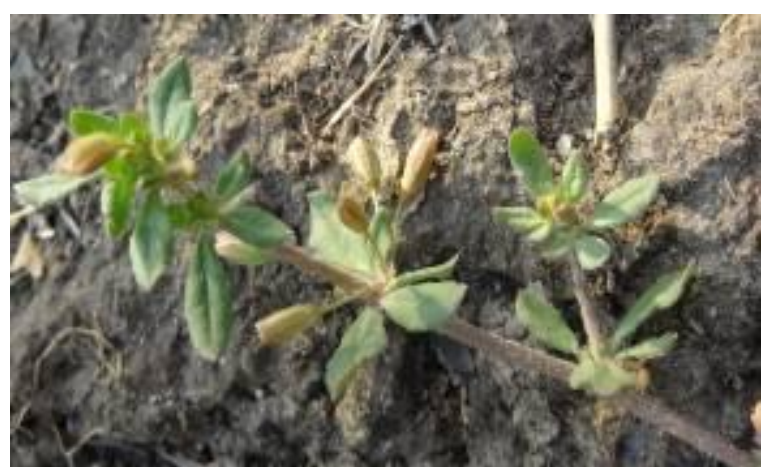

32) Mollugo pentaphylla 
After the struggle of intellectuals, scientists and others to form to survey and status of all the living organisms, by the assessment of IUCN. $(16,17,18,19)$ They gave some categories to plants. As per that IUCN status present enumerated plants given in Fig.No.2

In conclusion, if growing of ornamental plants as economic basis it is also useful, in the form of self employment schemes of various government or NGO organizations. To improve socio-economic status of who are living near to fort areas. But the anthropogenic activities on nature, when they minimize at narrow levels, then the biodiversity can be rooted to its ancient days.

\section{Acknowledgement}

The author grateful thank to Prof. S. Seeta Ram Rao, Professor, Department of Botany, Plant Physiology And Molecular Biology Lab.UCS, Osmania University, Hyderabad, Telangana.

\section{References}

1.Khoshoo, T.N. 1995. Biodiversity, Bioproductivity and Biotechnology. In: Farmers Rights and Plant Genetic Resources - Recognition and Rewards: A dialogue (eds) M.S. Swaminathan, Mac Millan India Ltd., pp.156-159.

2.BinuThomas, Rajendran, A., Aravindhan, Vand Maharajan, M. 2011. Wild ornamental chasmophytic plants for rockery. J. Mod. Biol. Tech., 1(3): 2021.

3.Jayanthi Palniswamy, Rajendran Armugum. 2014. Exploration of wild ornamental flora of Madhukkarai hills of South Western Ghats. Biolife I. J. O. L. S., Vol.2(3) pp.834-840.

4.Mayers, N. 1988. Threatened biotas:
Hotspots in tropical forests. Envi., 8: 187-208.

5. Rajagopal Reddy, S., Madhusudhana Reddy, A., Yasodamma, N. 2012. Exploration of wild ornamental flora of YSR District, Andhra Pradesh. Indian J. Fund. Appl. Life Sci., Vol. 2(1) pp.192-199.

6. Aasati, B.S., Yadav, D.S. 2010. Diversity of horticultural crops in North Eastern region. ENVIS Bull. Himal. Ecol., 12(1).

7. Swarup, V. 1998. Ornamental horticulture. MacMillan Indian Limited, New Delhi.

8. Joothimani Krishnaswamy, Rajendran Armugum, Sarvalingam Ariyan. 2014. Ornamental aquatic and semi aquatic plants in Coimbatore district. Biolife I. J. O. L. S., Vol.2(1) pp.557-570.

9. Naqvi, A.H. 2001. Flora of Karimnagar District, Andhra Pradesh, India. Ph.D. Thesis. Kakatiya University, Warangal, India.

10. Gamble, J.S., Fischer, C.E.C. 19151936. Flora of the Presidency Madras. Vols.1-3. Adlard \& Co. London (Reprinted 1957). Botanical Survey of India, Calcutta.

11. Hooker, J.D. 1872 - 1897. The Flora of British India. Vols. 1-7. L. Reeve Company, A. ford, Kent, USA.

12. Pullaiah, T., Chennaiah, E. 1997. Flora of Andhra Pradesh: India. Vol. 1. Ranunculaceae-Alangiaceae Scientific Publ. 1997.

13.Pullaiah, T., Prasanna, P.V., Obulesu, G. 1992. Flora of Adilabad District, Andhra Pradesh, India CBS Publishers \& Distributors-1922.

14. Jain, S.K., Rao,R.R. 1977. A hand book of field and herbarium methods. Today and Tomorrows Printers and Publishers, New Delhi.

15. Jochen Schaumburg, Christine Schranz, Julia Foerster, Antje Gutowski, 
Gabriele Hofmann, Petra Meilinger, Susanne Schneider, S., Ursula Schmedtje. 2004. Ecological classification of macrophytes and phytobenthos for rivers in Germany according to the Water Framework Directive Limnologica. 34: 283-301.

16.http://www.iucnredlist.org/technicaldocuments/categories-and-criteria.

17.IUCN. 2001. IUCN Red List Categories and Criteria: Version 3.1. IUCN Species Survival Commission. IUCN, Gland, Switzerland and Cambridge,
U.K. ii +30 pp.

18.IUCN. 1998. Guidelines for Reintroductions. Prepared by the IUCN/SSC Re-introduction Specialist Group. IUCN, Gland, Switzerland and Cambridge, U.K.

19.IUCN. 2003. Guidelines for Application of IUCN Criteria at Regional Levels. Version 3.O. IUCN Species Survival Commission. IUCN, Gland, Switzerland and Cambridge, UK. ii + $26 \mathrm{pp}$.

\section{How to cite this article:}

Odelu, G. 2016. Aquatic and Semi Aquatic Ornamental Flora of Karimnagar District, Telangana, India. Int.J.Curr.Microbiol.App.Sci. 5(3): 82-92. doi: http://dx.doi.org/10.20546/ijcmas.2016.503.012 